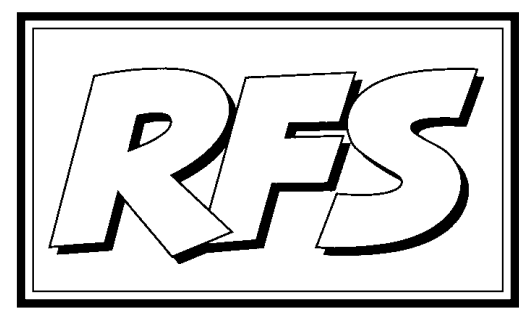

Revista de Fomento Social, 56 (2001), 433-459

\title{
La planificación en las ONGD ${ }^{1}$
}

Ma J OSé MONTERO SIMÓ²

\section{Introducción}

Las Organizaciones No Gubernamentales para el Desarrollo (ONGD) se conforman como un agente de gran valor para la sociedad civil, dada la labor que desarrollan. Pero esta misión no las exime de optimizar los recursos de los que disponen.

En una organización sin fines de lucro, ese límite mínimo no existe... pero sí existe la tentación de restar importancia a los resultados, de alegar: estamos sirviendo una buena causa, estamos haciendo la obra de Dios, estamos haciendo algo para mejorarle un poco la vida a la gente, y eso es de por sí un resultado. Eso no basta. Si una empresa

1 El presente artículo es una versión modificada de parte del capítulo «El Proceso de Planificación en las ONGD» de la tesis doctoral elaborada por Ma J osé Montero Simó y dirigida por Jesús N. Ramírez Sobrino, con el nombre de «El Marketing Social: una aplicación al caso concreto de Manos Unidas, Campaña contra el Hambre».

2 Profesora del área de Comercialización e Investigación de Mercados del Departamento de Gestión Empresarial y Métodos Cuantitativos de la Facultad de Ciencias Económicas y Empresariales - ETEA de la Universidad de Córdoba. La autora agradece a su director de tesis todo el apoyo, observaciones y sugerencias recibidas, sin todo lo cual no hubiera sido posible el desarrollo de trabajos como éste. 
malgasta sus recursos en algo que no da resultado, por lo general pierde su propio dinero. En cambio, una institución de este tipo pierde dinero ajeno: el de los donantes ${ }^{3}$.

Con el afán de mejorar el desempeño de su trabajo, optimizando los recursos disponibles, se ha elaborado este artículo. Donde se pone de manifiesto como, a pesar de las mejoras que se consiguen con el pensamiento estratégico, es necesario superar una serie de dificultades iniciales con las que se encontrará la ONGD. Y, cómo, una vez superadas estas barreras, convencidos sobre el interés de la planificación, antes de comenzar el proceso en sí, es conveniente considerar una serie de elementos claves: tener claramente definida y compartida la identidad corporativa y, más concretamente la personalidad corporativa, y contar con la información necesaria. Todo esto permitirá establecer las bases necesarias para desarrollar adecuadamente la labor de las ONGD en el Norte, fundamentándose en la filosofía del Marketing sob re la culminación satisfactoria de relaciones de intercambio.

\section{La filosofía del Marketing, inspiradora para la planificación en las ONGD}

En 1969, con base en la propuesta de Kotler y Levy ${ }^{4}$ se origina la corriente de pensamiento que defiende la aplicación del Marketing a todo tipo de organizaciones. Es entonces, cuando surge el concepto de Marketing sustentado en el intercambio de valores entre dos partes. Siguiendo esta línea se rompe con la versión restringida que limitaba el Marketing al campo de las transacciones comerciales, extendiéndose su aplicación a todo tipo de bienes 0 entidades que puedan ser objeto de intercambio, tales como ideas, actitudes, sentimientos, etc.

Marketing es un proceso social y de gestión a través del cual los distintos grupos e individuos obtienen lo que necesitan y desean, creando, ofreciendo e intercambio productos con valor para otros 5 .

La esencia de una relación de intercambio se establece en la entrega de valor. Resulta fácil concebir y perfilar relaciones de intercambio en el seno del mundo empresarial. Sin embargo, cuando se hace el intento en el campo social no resulta tan sencillo. La mayor dificultad no se presenta en la

\footnotetext{
3 P. Drucker (1990), p. 141.

4 P. Kotler y S. Levy (1969).

5 P. Kotler, D. Cámara e I. Grande (1995), p. 6.
} 
identificación de las partes sino, más bien, en la de la oferta de valor y la contraprestación, también con valor, que se deriva.

FIGURA 1

\section{Relaciones de intercambio de una ONGD}

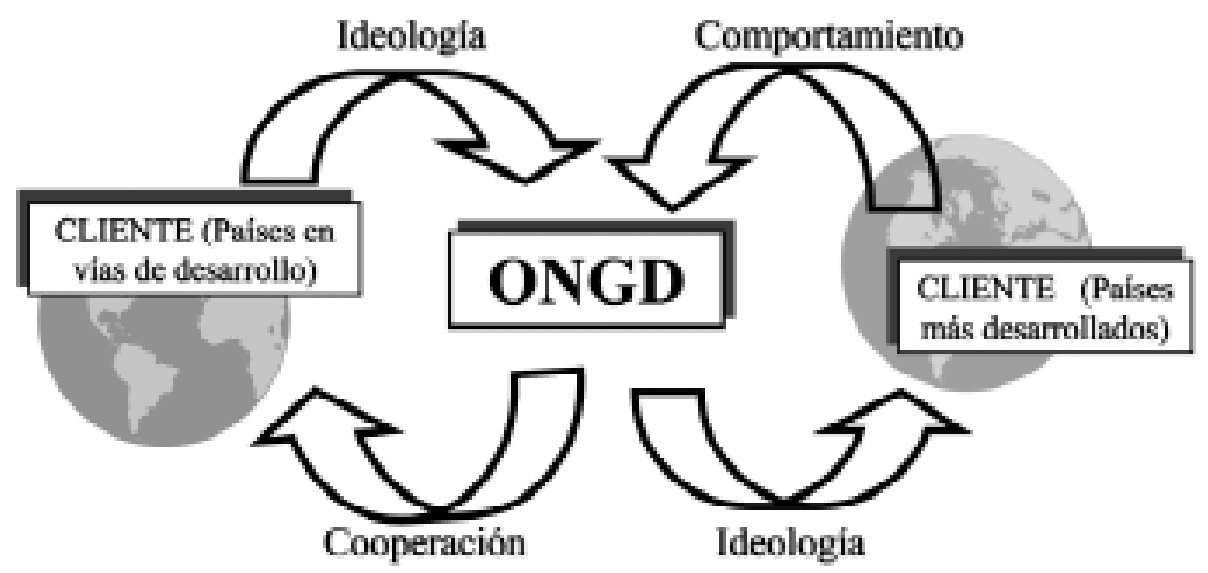

Fuente: Elaboración propia.

La figura 1 muestra una doble relación de intercambio en la que la ONGD desempeña un papel central, como eslabón clave, nexo entre los distintos actores. En el análisis de los flujos o intereses que tienen lugar en esta relación, lo primero que se plantea es la constatación de la existencia de un problema social, que atañe a todo el planeta y la vía para su solución es el establecimiento de un Nuevo Orden Internacional. Para ello, se constituye una ONGD que persigue esta causa social, analizando en primer lugar las necesidades de los países en vías de desarrollo ${ }^{6}$. De este análisis, se obtiene la ideología que, partiendo del Sur, se transmite a los países más desarrollados esperando la respuesta del cliente. Ésta se materializa en los comportamientos a través de los cuales se manifiesta la cooperación, vía de solución

6 Existen muchos términos que se emplean para denominar a estos países en contraposición con los países más desarrollados; dejando al margen la dialéctica abierta al respecto, se van a emplear las denominaciones de países del Sur y del Norte por la facilidad en su expresión. 
del problema social contemplado. Comportamientos que no hacen referencia exclusiva a la entrega de dinero, es decir, el habitante del Norte no se percibe exclusivamente como donante o fuente de financiación para el desarrollo de proyectos en el Sur.

La ONGD establece dos tipos de intercambios en su labor, limitándose el objeto de estudio del presente trabajo a la relación que tiene lugar con los países más desarrollados. De esta forma queda al margen del estudio la concepción de desarrollo que, atendiendo a las necesidades del Sur, se transmita por parte de la ONGD. Por tanto, los resultados favorables que resulten de la aplicación de la filosofía del Marketing, tal y como aquí se analizarán, repercutirán exclusivamente sobre la consecución de los objetivos planteados por la ONGD en su labor con los países del Norte, sin considerar las repercusiones que éstos tengan sobre la relación con el Sur. Aunque en todo momento se defiende la necesidad de establecer, con carácter previo a la labor en el Norte, la definición de desarrollo por la que se apuesta, bajo el planteamiento de los países del Sur.

Bajo el supuesto de la relación de intercambio entre ONGD y países del norte, en aras de optimizarla, la planificación estratégica se constituye como un elemento indispensable que permitirá concebir la relación para que exista, de la mejor forma, una entrega de valor en ambos sentidos. Así, en este trabajo se persigue exponer, bajo la perspectiva del Marketing, el proceso de planificación necesario para las ONGD. De manera que se consiga maximizar la consecución de los objetivos perseguidos por la ONGD en el Norte.

\section{Pros y contras de la planificación en una ONGD}

La planificación es simplemente una forma sistemática de que una organización intente controlar su futuro. Un plan es esencialmente una declaración de lo que la organización espera alcanzar, cómo hacerlo y cuando lograrlo. Virtualmente cada gerente reconoce la importancia de la planeación, porque la lógica implícita es innegable. En la práctica, sin embargo, a menudo la planificación no tiene lugar. Una razón para esto es que, los resultados son por lo general de largo plazo, y la alta gerencia da mayor importancia a los resultados inmediatos. Otra razón es que, por estar bajo considerable presión de tiempo, la gerencia intermedia está más orientada hacia la acción que a la planificación ${ }^{7}$.

Antes de exponer el proceso de planificación que debe seguir una ONGD, se han de considerar, de forma general que, a pesar de las ventajas que

7 J. P. Guiltinan y G. W. Paul (1994), p. 14. 
implica la planificación para este tipo de organizaciones existen una serie de frenos. Resultando necesario que los miembros de la organización sean conscientes de que el proceso no va a ser fácil al principio.

La planificación formal consiste en tomar de antemano, de manera conscientey, a ser posible, explícita, un conjunto de decisiones, programando los medios materiales y humanos necesarios para su ejecución.

En el sector no lucrativo en general, y en el caso de las ONGD en particular, las ventajas percibidas en el sector lucrativo no se ven tan claras ya que a los frenos de tiempo y ausencia de resultados inmediatos, propios también del sector lucrativo, sele unen otros ${ }^{8}$. La idea más extendida en este sector sobre los planes suele ser la de archivarlos, actuando en el día a día sin concebir la planificación. Y, con carácter más general habría que mencionar la extendida convicción de que la planificación y el Marketing son cosas de las empresas, algo impropio para las ONGD. Las organizaciones que actúan en el campo social, no se perciben sometidas a la presión de la competencia, ni cuentan con fuertes incentivos que conduzcan al personal a la elaboración y desarrollo de una adecuada planificación.

En el campo social se sufre a menudo amnesia sobre todas las actividades llevadas a cabo en el pasado. No se dispone de datos sobre el pasado y la rotación del personal es elevada, dificultando la existencia de información del pasado entre los miembros de la organización.

Existe una dificultad añadida en estas organizaciones al plantearse la planificación. La necesidad de predecir las acciones de los competidores en el horizonte temporal, resulta impensable en el sector, teniendo en cuenta que, ni unos ni otros conciben tan siquiera su propia planificación. Además, es más difícil conceptualizar la competencia, en el entorno de estas entidades entran en juego competidores enmarcados en dos niveles muy diferentes, lo que se podría denominar, competidores «enemigos» refiriéndose a aquellos que inducen a comportamientos contrarios al deseado en la población objetivo y competidores «amistosos» que representan a las organizaciones similares. Este último tipo de competencia hay que tenerlo muy en cuenta, dadas las peculiaridades con las que cuentan, estos colectivos pueden ayudarse entre sí, pero también pueden fragmentar esfuerzos, solapamientos, crearse problemas u otro tipo de dificultades.

8 N. Bloom y W. D. Novelui (1981). 
La posibilidad que aporta la planificación de establecer estándares de desempeño que permiten medir los resultados, presenta mayores dificultades para alcanzarse en el campo social que en el lucrativo. Resulta más complejo establecer objetivos específicos y cuantificables dado que se está trabajando sobre una idea, comportamiento o actitud, lo que conlleva la dificultad de definir las medidas para evaluar el grado de cumplimiento de dichos objetivos.

En el campo social, se parte de una escasa e inadecuada planificación, a lo que se suma la ambigüedad de los objetivos planteados, resultando por tanto, prácticamente imposible determinar la relación directa entre las causas -acciones planificadas-, y los efectos -consecución de objetivos-.

Por último, se debe indicar que en este sector se sufre un recorte presupuestario fuerte, por lo que emplear recursos económicos y humanos en este tipo de actividades, en las que no se perciben beneficios inmediatos, no es considerado apropiado. La planificación se percibe como un gasto, sin contemplar que ese gasto y esfuerzo inicial repercutirá sobre un ahorro importante en costes, tiempo y esfuerzo a la larga.

En cualquier caso, la planificación estratégica no es la solución a todos los problemas y dudas que plantea el funcionamiento de una organización. Tampoco es la panacea, ni el sustituto del liderazgo, sino que se configura como una herramienta de apoyo para la dirección de la organización. Además, no en todos los casos se llevará hasta sus máximas consecuencias, dependiendo todo de la situación que atraviese la organización.

A pesar de los frenos y limitaciones existentes, creemos queda justificada la conveniencia de la planificación en una ONGD. Algo que ya se viene desarrollando, con éxito, en el seno de algunas de ellas.

Cuando se hace referencia a la planificación se trata de algo más que la mera elaboración de planes escritos, que persiguen alcanzar unas metas previamente establecidas; no se puede ignorar la incidencia del pensamiento en la estrategia o dirección básica del negocio en el que se está. Tampoco se ha de pensar en algo tan majestuoso que requiera deun departamento propio de planificación; es mejor concebir un enunciado simple de la estrategia, la definición de unas pocas áreas clave de resultado, y un conjunto de objetivos organizacionales sencillos.

En ningún momento se parte de un conocimiento cierto del futuro, lo que conduce a la ONGD a necesitar algo más que planificar; se necesita un 
proceso de pensamiento que, más que basarse en un enfoque determinista, apunte a la explotación de las oportunidades que se puedan presentar. Se ha de pensar en un proceso de estrategia de acción, versátil, adaptativo y que permita y facilite la capacidad de reacción; contando con medidas de los resultados o indicadores críticos que faciliten calibrar la efectividad de las estrategias de acción desarrolladas. No debe existir tanta preocupación por el éxito o fracaso como por la capacidad de adaptación continua a los efectos de las estrategias seguidas. La planificación, tal y como se entiende en su acepción más simple, se ha de complementar con el proceso de decidir cómo comportarse sobre la base de lo que sucede en el ambiente inmediato y en el futuro más próximo de la organización, entendiéndose como planificación la traducción de esas decisiones en acciones manejables. Todo este proceso se apoya en una comprensión clara de quiénes somos realmente, de qué es lo que somos capaces de hacer, de cuál es el negocio en el que estamos, de qué valor creamos para nuestros clientes, y de cómo nos diferenciamos para conseguirlo y conservarlo, es decir, se apoya en la gestión de la identidad corporativa.

Por tanto, el proceso de planificación en el ámbito corporativo la ONGD debe partir de la formulación de las siguientes preguntas: ¿quiénes somos? y ¿qué hacemos? Para, posteriormente, plantearse en qué situación está, en qué lugar y en qué momento se encuentra, para poder decidir dónde quiere llegar y de qué manera conseguirlo. Éste es el proceso de planificación que, como se ha indicado, se debe considerar como un proceso activo, flexible y que persigue, en última instancia, promover el pensamiento y la acción estratégica en el seno de la organización.

\section{Elementos claves para planificar en las ONGD}

Tal y como se ha indicado, existiendo la necesidad de contar con un proceso de pensamiento, se establecen como elementos claves para desarroIlarlo: definir claramente la identidad corporativa y, más aun la personalidad de la ONGD, y disponer en todo momento de la información necesaria.

\subsection{Identidad e imagen corporativa}

El proceso de planificación corporativa tiene que partir del establecimiento previo de la identidad corporativa. Esta servirá de guía u orientación a lo largo de todo el proceso, estableciendo todas las bases sobre las que trabajar. 
La imagen es uno de los principales activos con el que puede contar una organización, puesto que ayuda a determinar el comportamiento de una persona con respecto a la misma, facilitando la fidelidad de sus voluntarios y donantes e impulsando un espíritu de bloque entre sus colaboradores. Pero, para que efectivamente sea un punto fuerte debe reunir algunas condiciones. En primer lugar, la imagen corporativa debe ser una síntesis de la realidad de la organización, y reflejar fielmente la cultura y los valores que allí se viven, y esto no sólo para que sea creíble, sino también para que sea defendible. Por tanto, es necesario adaptar permanentemente el mensaje corporativo a los cambios estratégicos de la empresa. En segundo lugar, ha de ser singular y exclusiva, de forma que facilite la identificación de la organización. En tercer lugar, ha de ser eficaz: notable y notoria. Por último, ha de ser coherente, opuesta a la dispersión. Así, la imagen corporativa tiene que ser una variable estratégica: un valor a largo plazo; duradera y coherente, - un fiel reflejo de la marcha de la organización-, y ser una consecuencia de considerar a la organización como un todo ${ }^{9}$

A partir de ahora al hablar de imagen corporativa se hará referencia al retrato de una organización, tal y como lo perciben los distintos públicos objetivo. Mientras que la identidad corporativa será la forma en que la organización se presenta ante dichos públicos. Hablar de imagen o identidad no tendrá mayor repercusión, ya que sehan de considerar como las dos caras de un mismo espejo. Dependerá desde donde se mire, pero siempre se hará referencia a una misma realidad.

Las dos caras del espejo no siempre coincidirán. Puede ser que la imagen corporativa, tal y como se percibe en el exterior, no sea un fiel reflejo de la identidad corporativa de la organización. Existen multitud de factores que influyen en el proceso de percepción. Existen factores personales y ambientales decisivos sobre la percepción de los distintos públicos objetivo.

Teniendo presente esta correspondencia entre las dos caras se van a analizar los elementos y la gestión de la identidad corporativa, tal y como se percibe desde el seno de la ONGD. Se trata de una serie de elementos interrelacionados y que como tal han de gestionarse. A continuación, van a analizarse cada uno de ellos para poder comprender mejor su valor y sentido dentro de la identidad corporativa.

- La propia personalidad de la organización. La personalidad corporativa se compone, básicamente, de la misión, visión, valores e historia de la ONGD. Constituye la manifestación de la autopercepción de la organización, incluyendo las intenciones y la forma de reacción ante los distintos estímulos. Los medios con los que cuenta una organización

9 J.L. Martínez Sánchez (1998 a), p. 27. 
para transmitir la personalidad de la organización tanto en el ámbito interno como externo, siguiendo su filosofía, son: comportamientos, comunicación y simbolismo.

- Los comportamientos. Los comportamientos conforman el medio más importante y eficaz a través del cual se crea la identidad corporativa de la organización. Los públicos objetivo juzgan a la organización, en última instancia mediante la valoración de las acciones desarrolladas por ésta. Esta variable que parece la más sólida, puede ser directamente influenciada por la comunicación y/o simbolismo, mediante la concesión de mayor o menor importancia a determinados aspectos. Esta variable junto a la personalidad corporativa configuran lo que muchos autores vienen a denominar como la cultura de la organización.

- La comunicación corporativa. La comunicación actúa como el instrumento más flexible para configurar la identidad corporativa, por tanto suele utilizarse tácticamente, con rapidez.

- Los símbolos empleados por la organización. El simbolismo aparece como la herramienta armonizadora, ofreciendo una indicación implícita de lo que representa la organización o por lo menos de lo que desea representar.

\subsubsection{Gestión de la identidad corporativa}

Todos estos elementos no pueden abandonarse, ha de trabajarse sobre ellos. Para que la identidad se transmita de forma eficaz y duradera generando una imagen positiva en la mente del individuo, debe cumplir tres requisitos ${ }^{10}$ : Debe basarse en la propia realidad de la ONGD, descartando por completo la pretensión de inducir «imágenes de laboratorio». En segundo lugar, la síntesis que supone la identidad corporativa debe hacer prevalecer los puntos fuertes de la organización, para lo cual es necesaria una estrategia integral y programada. En último lugar, la gestión de la identidad exige una coordinación de las políticas formales con las funcionales. Entendiendo, por políticas formales los elementos que se han señalado como componentes de la identidad corporativa, resulta ingenuo pensar que éstos son o pudieran ser los generadores exclusivos de la imagen corporativa.

$10 \mathrm{~J}$. Villafañe (1993), pp. 30 y ss. 
De esta forma se entiende que de poco sirve una gestión rigurosa de los activos intangibles que componen la identidad corporativa si las políticas funcionales -comercial, financiera, recursos humanos, etc.- de la organización se gestionan independientemente impidiendo un desarrollo global que persiga sinergias y optimice los resultados en términos de imagen corporativa. Siendo consciente de esta visión global y coordinada, una organización tiene que conocer claramente cuál es el elemento aislado de la organización más importante en la creación de la identidad propia, pudiéndose tratar éste, incluso, de cualquier política funcional de la organización. Es decir, cualquier organización que cuente con una identidad fuerte tendrá necesariamente un aspecto o elemento clave diferenciador.

Ahora bien, si se presta atención a las empresas de servicios, se ha comprobado, lo cual parece lógico, que dada su fuerte dependencia del personal de primera fila, el comportamiento de estos miembros es el que suele actuar como elemento diferenciador. Parece configurarse como el aspecto que puede otorgar una mayor ventaja diferencial, sobre todo si la organización es la primera que avanza en este sentido. De tal forma que las ONGD tienen en este terreno importantes vías de desarrollo y de mejora para la consecución de sus objetivos. Es un campo poco explotado en este tipo de organizaciones, sobre todo desde la perspectiva de la mejora de la atención al cliente.

De todo lo expuesto se ha de extraer como conclusión que la identidad corporativa confiere sentido a cualquier pensamiento, decisión o acción que tenga lugar en el seno de la organización, a la vez que se retroalimenta de todos y cada uno de estos elementos.

Se van a abordar, con detalle, elementos de la personalidad corporativa, elementos fundamentales, sobre los que se sustenta toda la proyección de la ONGD. Sirviendo, por tanto, de punto de partida en el proceso de planificación.

\subsubsection{La personalidad corporativa}

Los elementos que componen la personalidad corporativa son el eje fundamental sobre el que se ha de vertebrar la organización. La historia de la ONGD se va construyendo con el paso del tiempo. Pero la misión, visión y valores han de ser definidos explícitamente y compartidos por todos los miembros de la ONGD. Por ello, los tres elementos se tratan de forma aislada 
pretendiendo poner de manifiesto su importancia y como tratarlos de forma interrelacionada para poder planificar mejor.

Una ONGD puede contar con una definición de misión explícita desde hace mucho tiempo, pero quizás al plantearse la planificación, sea el momento de revisar el contenido y el enfoque de la misma. En cualquier caso, la organización contará con una misión implícita, que una vez se haya decido planificar necesitará formularse expresamente.

Para nosotros el elemento clave es la misión. Toda institución nace con ansias de perpetuar su acción. Así, una organización ineficaz está condenada a morir. Pero una ONGD nace para cumplir con una misión, que trasciende la mera maximización del beneficio. Siempre y cuando la organización cumpla con la tarea encomendada dentro del sistema social se justifica su existencia, pero cuando la misión se agota o instrumentaliza, la organización debe redefinirse o replegarse. De este modo nos parece que el término que mejor las define es el acuñado por Mintzberg, que las denomina «Organizaciones Misionarias»: no son sólo organizaciones con misión, sino entidades que se configuran de tal forma que la única posibilidad de desarrollo es satisfacer el motivo por el que fueron creadas. Así podemos afirmar que el cumplimiento de la misión es un imperativo ineludible para este tipo de entidades. La misión define el propósito, el motivo de existencia de la organización. Sin este principio organizacional, la institución no lucrativa podría verse emprendiendo actividades para las cuales no está capacitada. El concepto de misión como fuerza conductora permite a la organización decidir sobre la orientación de sus servicios y sobre el tipo de población objetivo a la que dirigirá su acción ${ }^{11}$.

La misión da respuesta en gran medida a las preguntas de quiénes somos y qué hacemos, o dicho de otra forma en qué negocio estamos. Ahora bien, un correcto enunciado de la misión contempla las fortalezas de la organización, pero siempre en consonancia con las oportunidades del entorno y debe comprometer a todos los miembros de la organización a la acción, al trabajo por esa misión. Es decir, tal y como Peter Drucker indica12, debe contemplar tres aspectos: oportunidades, competitividad y compromiso. Se deberá hacer mejor lo que ya se sabe hacer, y que además permita distinguirse, diferenciarse de los demás, siempre que los miembros de la organización crean en ello y se comprometan en su desarrollo. Para conseguir un enunciado efectivo de la misión, los elementos esenciales, competitividad y desarrollo de oportunidades, se pueden enunciar considerando los siguientes aspectos:

\footnotetext{
11 J.L. Martínez (1998 b), p. 71.

12 P. Drucker (1990), p. 15.
} 
- El cliente. No se define en términos de algún segmento del mercado o categoría estadística, sino en función de una premisa de necesidad básica y definitoria que lleva a esa persona o entidad a considerar la posibilidad de hacer negocios con nuestra organización.

- La premisa de valor. Se define, no en los términos de lo que nuestra organización hace, fabrica, vende o entrega, sino en función del valor fundamental que representa como satisfacción de la premisa de la necesidad del cliente.

- Lo que nos hace especiales. Nuestro medio especial de crear valor, para obtener y conservar el interés del cliente ${ }^{13}$.

Dar respuesta a la pregunta ¿quiénes somos? conduce además, ineludiblemente a examinar detenidamente cuál es la identidad propia de la organización. La misión, por tanto, es el equilibrio entre las oportunidades existentes y las fortalezas e identidad propia de la organización. La búsqueda de ese equilibrio implica el análisis y diagnóstico de la situación para detectar las oportunidades y fortalezas, estudio que ha de complementarse con el de la identidad corporativa.

Para definir la misión, en el estudio de la identidad corporativa, hay que prestar especial atención a los valores de la organización. La determinación de los valores centrales de la organización lleva, en muchas ocasiones a los directivos, a enumerar un conjunto de valores carentes de sentido para la institución, que sólo representan una lista de valores ideales para la sociedad. Esta situación implica, casi siempre, perder la valiosa oportunidad que tienen los directivos de prestar atención y otorgar significado a lo que hace el personal de su organización, algo fundamental para conseguir el compromiso que se necesita con la formulación de la misión. Ya que los valores son las creencias morales en las que se basan to das las actuaciones de la ONGD.

La misión se conforma por tanto como la razón de ser de la organización, su correcta formulación, como ya se ha indicado, además de conseguir comprometer al personal ${ }^{14}$, implica la existencia de un elemento diferencia-

13 K. Albrecht (1996), p. 171.

14 Según F. Iríbar Bilbao (1996), p. 25, «La misión permite que todos los colaboradores de la organización, ya sean profesionales, donantes o voluntarios, puedan reconocer su contribución personal a la tarea fundamental de la organización», lo que les permite alcanzar un mayor nivel de compromiso con ésta. 
dor y de un determinado nivel de competitividad. Todo esto permite a la organización determinar quién es su competencia en el ámbito de las necesidades que cubre y de la categoría de productos que ofrece.

Al analizar algunas ONGD nos encontramos con definiciones explícitas de su misión bastante recientes; aquí se presenta el caso concreto de Intermón y Manos Unidas:

- La Misión de Intermón es: Contribuir a generar cambios que hagan posible el desarrollo sostenible de los países del Sur y que permitan alcanzar unas estructuras sociales justas en las relaciones entre los pueblos y fomenten, al mismo tiempo, una cultura de solidaridad.

- La Misión o finalidad de Manos Unidas es: la lucha contra el hambre, la mala nutrición, la miseria, la enfermedad, el subdesarrollo y la falta de instrucción; y contra sus causas: la injusticia, el desigual reparto de los bienes y las oportunidades entre las personas y los pueblos, la ignorancia, los prejuicios, la insolidaridad y la insensi bilidad. El objetivo de su trabajo es contribuir al desarrollo integral de los países del Sur, tomar parte activa en la lucha contra el hambre y la desigualdad y apoyarles en su proceso de liberación de la miseria y la dependencia.

De la observación de estos enunciados de misión se deduce la falta de una definición precisa de los tres elementos esenciales comentados. En el enunciado se ignora al cliente del Norte. No existiendo, por tanto, ninguna oferta de valor para ellos. En cuanto al cliente del Sur, la misión refleja una premisa de valor pero poco específica o diferenciada. En cuanto a los tres elementos implícitos de una misión según los define Drucker, en estas formulaciones podría existir el elemento de compromiso, pero en cualquier caso estarían ausentes el elemento de competitividad, en cuanto a comunicar las fortalezas e identidad propia con un marcado carácter diferencial, y el elemento conformado por las oportunidades detectadas.

Es importante recalcar la ausencia de una propuesta de valor para el cliente de los países del Norte en la definición del negocio de estas organizaciones; es importante que la ONGD no olvide la existencia de una doble relación de intercambio, que no puede tener lugar si una de las partes no quiere. Es necesario que en su misión, la organización considere las necesidades que va a cubrir en estos países, y la forma en que va a hacerlo con carácter diferencial y competitivo en el mercado. En estas formulaciones no se percibe referencia alguna a la población objetivo del Norte, quedan 
totalmente al margen de la definición de su misión, por tanto, si no van a ofrecerles nada nos preguntaríamos ¿por qué la ONGD pretende establecer una relación con ellos?, a la vista de su misión, ellos no forman parte de su negocio, 0 al menos no lo hacen con carácter primordial.

Es útil que, para formular la misión por primera vez o para reformularla, la ONGD se plantee preguntas como las que siguen, observando el equilibrio entre éstas y la misión:

- Misión del negocio: ¿En qué negocio está?

- Definición del cliente: ¿Quién es su cliente?

- Necesidades objetivo: ¿Qué necesidades intenta satisfacer?

- Mercado-meta: ¿En qué segmento de mercado quiere centrarse?

- Identificación competencia: ¿Quiénes son sus mayores competidores a nivel de necesidades y de categoría de productos?

- Posicionamiento: ¿Qué beneficios competitivos o ventaja diferencial quiere ofrecerle a su mercado meta?

En cuanto a la visión, hay que entender que, contempla la misión, pero se centra en la autopercepción de la organización en un entorno favorable, mientras que la misión es una formulación más racional. La visión engloba también en cierta medida a los valores, la filosofía de la organización, sus objetivos y estrategias, y los elementos clave de diferenciación. De esta forma, la visión debe actuar como factor de motivación para todo el personal de la organización. La visión ha de ser un enunciado motivador que indique dónde quiere llegar la ONGD.

El proceso de planificación estratégica se halla directa e ineludiblemente vinculado a la formulación previa de la misión, la visión y los valores, elementos característicos de la personalidad de la organización que no se reformulan constantemente, pero que resultan indispensables para poder reflexionar sobre la estrategia a seguir. Si no están claramente definidos antes de empezar con la planificación, hay quehacerlo, acudiendo al desarrollo de todas las reflexiones o análisis necesarios para lograr su formulación. De aquí se deduce el valor de la información, desde que se establecen las bases del proceso de planificación. Siendo necesaria, de igual forma, a lo largo de todo el proceso. 


\subsection{El valor de la información}

La información es un elemento necesario para una ONGD, con un valor inestimable y que, por tanto, ha de mantenerse de forma activa, resultando el eje central sobre el que se vertebra todo el proceso de planificación tal y como se expone a continuación.

Las compañías encaran hoy día un tremendo reto: convertir sus montañas de datos en información clave para los que toman decisiones. La verdadera competitividad de una organización se determina por la capacidad que demuestran sus empleados decidiendo a tiempo, lo más adecuado para mejorar continuamente la rentabilidad ${ }^{15}$.

Las ONGD también actúan en este entorno cambiante e incierto en el que la información juega un papel crucial, por tanto deben detectar y analizar sus necesidades de información, para poder darles respuesta, siempre que se desee alcanzar los objetivos organizacionales planteados.

Una de las grandes partidas que debe existir en una ONGD, como objetivo de información, ha de ser todo lo relativo al conocimiento del adoptante objetivo o cliente del Norte. En concreto, se ha de captar información del adoptante relativa a las necesidades, deseos, móviles, situación personal, capacidad de cara a la adopción del comportamiento, el proceso de adopción seguido y los factores del macroentorno determinantes. Esta información no sólo es una partida importante, sino que, además, debe ser el comienzo de la labor en el Norte bajo la perspectiva del Marketing considerando la existencia de una relación de intercambio entre ONGD y adoptante objetivo. Se trata del primer paso que debe dar la organización, antes de diseñar su oferta, incluso su propia identidad. Para ello, la ONGD debe contar con las herramientas necesarias para captar, tratar y mantener viva la información.

La gestión de la información para el fin señalado supone, primeramente, la identificación concreta de las necesidades informativas que surgen de las decisiones clave a tomar. Al mismo tiempo será necesario conocer al detalle cuál es la información disponible, cuál es la información que se cree conocida, y cuál es la información que se pudiera tener. De la contrastación entre necesidades y los datos que se tienen en la organización, se identifican las deficiencias informativas. Si se comparan esas mismas necesidades con lo que la ONG cree que conoce, se localizan informaciones que será preciso matizar o verificar. Y por último, si se tiene en cuenta la información que fuera posible conocer, se precisaría el grado de factibilidad de cubrir las necesidades identificadas.

La gestión de las deficiencias, matizaciones, y verificaciones informativas que son factibles realizar en una organización conlleva el desarrollo de las siguientes activida-

15 M. Martín Dávila, J. Manera Bassa y E. Pérez del Campo (1997), p. 111. 
des: el acceso a una pluralidad de fuentes informativas, el empleo de un conjunto de herramientas para la captación y tratamiento de datos, la distribución de la información a los decisores, y la verificación del valor añadido que implica su uso en términos de prestancia.

La sucesión fluida de estas acciones interrelacionadas, y la conjunción armónica de la multiplicidad de actores implicados demandan algún esquema de sistematización de este proceso. El cuerpo teórico de las técnicas tradicionales proporciona diferentes herramientas como soluciones alternativas a esta necesidad gerencial. De todas ellas, la rama del Marketing aporta un instrumento que cubre con total solvencia y garantía los requisitos y condicionantes expuestos, es lo que se conoce como Sistema de Información en Marketing ${ }^{16}$.

Mantener información actual, relevante y precisa en el día a día resulta complejo para las ONGD, atendiendo a distintas razones, tales como: bajo presupuesto disponible; falta de una cultura organizacional fuertemente arraigada, en un entorno de trabajo establey firme en el tiempo, que genereuna memoria histórica; fuertes dificultades para captar información con marcado carácter cualitativo y subjetivo. Pero, en cualquier caso, resulta conveniente hacer el esfuerzo, disponiendo de información suficiente que aporte valor añadido a la gestión de las ONGD en el Norte, favoreciendo la planificación necesaria para conseguir mayor eficacia y eficiencia en su trabajo.

\section{El proceso de planificación estratégica}

Una vez considerados los elementos de valor para planificar, es decir, una vez alcanzado un acuerdo inicial, anterior al proceso propiamente dicho de planificación estratégica, contando con la información necesaria, se pasaría a otra fase en la que se determinará ¿dónde estamos? y ¿dónde queremos llegar? realizándose un análisis y diagnóstico de la situación en el ámbito corporativo para poder definir las prioridades estratégicas apoyándose en la visión de la organización.

Por último, se ha de indicar qué es lo que hay que hacer para conseguir alcanzar los objetivos propuestos, destacando la importancia de la existencia de una visión de futuro bien formulada y la existencia de un documento donde se recoja toda la reflexión desarrollada hasta el momento, dicho documento es lo que se conoce como Plan Estratégico.

Estas fases constituyen realmente el proceso de planificación. Proceso que se representa en la figura 2.

16 B. García Izquierdo (1996), p. 219. 
FIGURA 2

\section{Proceso de planificación estratégica}

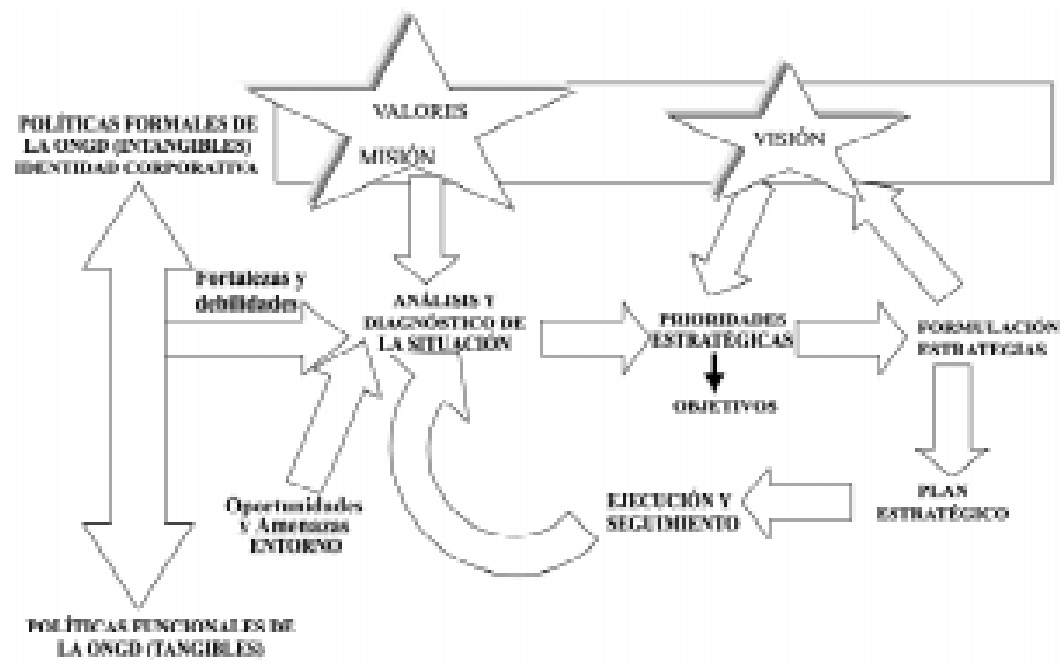

zQuiéns sostos LOUE HuCEMOS!

¿POWDE ESTAYTOS?

_DONDE QUEREMOS D.ECAR?

COONO LUEGR:

Fuente: Elaboración propia

Para comenzar con el proceso de planificación propiamente dicho, tal y como se ha mencionado, habría que realizar, en primer lugar, un análisis y un diagnóstico de la situación. Este análisis permite detectar las oportunidades y amenazas existentes en el entorno y las fortalezas y debilidades con las que cuenta la organización para afrontarlas.

El análisis del entorno implica examinar las tendencias, fenómenos 0 realidades que afectarán positiva o negativamente a la ONGD, o al sector no lucrativo en su totalidad. Para ello, es necesario tener clara la misión de la organización. Sin haber dado este paso resultaría muy complejo detectar claramente los límites dentro de los cuales el entorno ejercerá alguna influencia sobre la propia ONGD o sobre su competencia a los distintos niveles. Tener una buena definición de misión permite, en primer lugar, conocer directamente quién conforma el microentorno dela organización, es decir, facilita la identificación de: la competencia, de los clientes, proveedo- 
res, intermediarios o distribuidores y grupos de interés. En segundo lugar, permite detectar los factores del macroentorno que pueden afectar a la ONGD.

Una vez identificado el mercado, para estudiar al cliente es útil conocer el proceso de adopción de comportamientos que sigue, y conocer en conjunto todos los aspectos que influyen en la demanda de los productos ofertados por la ONGD: necesidades, deseos, móviles, situación personal y capacidad de adopción.

El adoptante objetivo, no se encuentra aislado, sino que está inmerso en un entorno en el que recibe una gran diversidad de ofertas, ofertas que dadas las restricciones de tiempo, capacidad física y dinero con las que cuenta el individuo, son excluyentes entre sí. Esta situación hace necesario considerar la competencia tal y como la percibe el cliente, si realmente se le quiere hacer llegar una propuesta concreta.

Para atender, realmente, a la interacción de este colectivo con la ONGD hay que mantener una actitud proactiva. Siendo necesario abandonar la actitud reactiva que generalmente se viene manteniendo en este tipo de organizaciones. Se han de evaluar las fortalezas y debilidades de la competencia para anticiparse a sus acciones. Dado que la organización satisfará, con su producto, las necesidades de los clientes mientras cuente con una ventaja competitiva que le permita asegurar el logro de sus objetivos en el largo plazo.

Deesta forma, hay que profundizar en un concepto de competencia mucho más complejo de lo que a primera vista puede parecer. El entorno competitivo de una organización esta compuesto por más factores que la mera existencia de las organizaciones directamente «rivales».

Hay que situarse en la óptica de un cliente para entender el proceso mental que sigue y así tener una visión completa de la competencia que percibe éste a lo largo del proceso de adopción. En primer lugar, el individuo se encuentra con una gran variedad de alternativas para satisfacer sus deseos concretos. El cliente cuenta con unos recursos limitados, sean del tipo que sean, y un elevado número de necesidades o deseos por satisfacer por lo que ha de renunciar a la satisfacción de algunos de ellos, estableciéndose por tanto un primer nivel de competencia. Posteriormente, se presentan ante los ojos del individuo una amplia gama de formas básicas para satisfacer sus necesidades, dando lugar a la aparición de otro 
nivel de competencia denominado genérico. En el siguiente nivel del proceso, aparecen diversidad de productos o formas de productos dentro de lo que hemos denominado formas básicas de satisfacción, situando por tanto al individuo ante un nivel de competencia de formas o productos. Por último, se le presenta un espectro de marcas donde tiene que elegir entrando a competir unas con otras.

Estos mismos pasos son los que ocurren en el proceso que sigue un cliente al analizar las distintas alternativas entre las que elegir hasta llegar a relacionarse con una ONGD. La siguiente figura presenta un ejemplo para ilustrar los distintos niveles de competencia en una ONGD. Aparecen destacados en negrita los distintos niveles de competencia de Manos Unidas en el caso de la campaña para la eliminación de la deuda externa.

\section{FIGURA 3}

\section{Niveles de competencia de una ONGD}

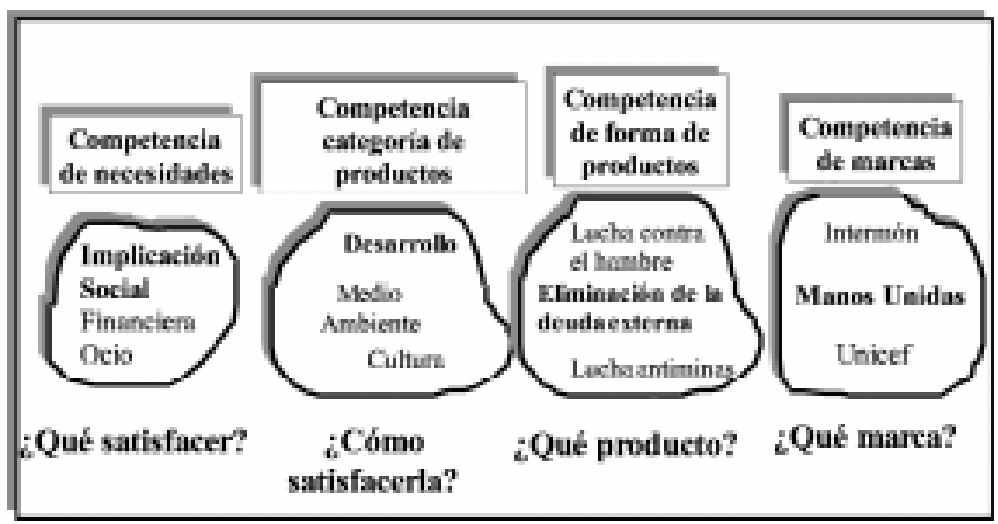

Fuente: Elaboración propia ${ }^{17}$.

Estos niveles de competencia, que parten de la visión del cliente, han de ser contemplados también en el seno de la organización en distintos ámbitos.

17 El empleo del término marca, dado el tipo de producto al que se hace referencia en este trabajo, donde la propia ONGD es el elemento más tangible, va a equipararse con lo que se denominaría estrictamente denominación social de la organización. 
En primer lugar, en el ámbito estratégico, cualquier organización ha de analizar con detenimiento los dos primeros niveles. Es decir, antes de nada, antes de comenzar cualquier actividad, se ha de ser consciente de la necesidad que se va a satisfacer y con qué categoría de productos se va a atender ésta. Se ha de estudiar qué necesidades compiten por los mismos recursos de los que dispone el individuo y una vez centrados en una necesidad concreta, ver qué categorías de productos compiten, nuevamente, para satisfacerla.

Una vez desarrollado este análisis con detenimiento, estratégicamente, en una segunda fase se procederá a analizar la competencia a nivel de formas de productos y marcas. Este estudio se circunscriben al ámbito del Marketing. Una vez que la organización en su conjunto tenga clara la necesidad que cubre y la forma genérica de hacerlo, los especialistas en Marketing se encargarán de investigar la competencia entre formas de productos y entre todas las marcas u organizaciones que ofrezcan esos mismos productos sociales. Cualquier acción de Marketing, en el seno de una ONGD, ha de diseñarse bajo estas consideraciones, teniendo en cuenta que el orden de selección que sigue el individuo a la hora de plantearse las alternativas de forma de productos y marcas puede variar.

En el caso de las ONGD la forma de percibir la competencia, sobre todo a estos dos últimos niveles, presenta una serie de peculiaridades; algunos autores hablan de «competencia amistosa». La realidad es que para un individuo con recursos-monetarios y no monetarios- limitados, las distintas alternativas son, en la mayoría de los casos, excluyentes entre sí. Aunque no se quiera emplear la palabra competencia por el componente de rivalidad que parece implicar, es necesario ser consciente de su existencia en la configuración mental del individuo. Esta ausencia de rivalidad expresa, existente en el terreno social, pone de manifiesto la necesidad de analizar y actuar de forma proactiva para fomentar la colaboración entre ONGD. Por tanto, se han de contemplar y promover acciones conjuntas compartidas, se ha de trabajar por la especialización de las ONGD en distintas formas de producto, en distintos segmentos de población, en las distintas fases a seguir hasta culminar el proceso de modificación de los comportamientos, etc. Sin confundirlo con inexistencia de alternativas excluyentes ante las que se sitúa el individuo.

En cuanto a los proveedores, para el caso de las ONGD, y en concreto en su relación con los países del norte, esta figura no tiene un peso relativo muy 
elevado, dado que se limita a la figura del proveedor de material de oficina y poco más, como ocurre en la mayoría de las empresas de servicios. Sin embargo, si se atendiera a la relación con el Sur, y en concreto, a las actuaciones de emergencia o puramente asistencialistas la figura del proveedor cobra mayor relevancia, ya que se trabaja con ingentes cantidades de bienes, de productos tangibles.

La figura del intermediario es vital también en el tipo de acciones, con los países del Sur, que se acaban de comentar. Sin embargo, en la relación con el Norte, actualmente esta figura apenas está definida como tal. A pesar de ello, dentro de este colectivo, bajo la perspectiva de la distribución del Marketing, como facilitadores del flujo del producto desde la ONGD hasta los clientes, y como agentes externos se podrían considerar algunas formas como las empresas, colegios, etc.

Por último, dentro del microentorno de las ONGD, cabe destacar el importante papel que cobran los grupos de interés, entendiendo como tal todo colectivo con impacto actual o potencial sobre la capacidad de la organización para alcanzar sus objetivos ${ }^{18}$. Resulta lógico que las ONGD les presten especial atención, dado que en la realidad estos colectivos ejercen una gran fuerza, enjuiciando cualquier acción desarrollada por las ONGD, pudiendo bloquear o favorecer cualquiera de sus actividades.

Se debe trabajar en las relaciones con estos grupos, para generar un ambiente favorable para el establecimiento del intercambio entre las partes. Para ello, básicamente se cuenta con la herramienta de las relaciones públicas, siendo necesario emplearla en todos los niveles de la organización y no sólo desde el departamento de marketing o de comunicación. Entre estos grupos de presión, las ONGD se pueden encontrar esencialmente con: los medios masivos de comunicación, a través de la emisión de noticias; la sociedad, a través de los distintos movimientos populares; determinadas instituciones de carácter público; y grupos de interés empresariales que según sus propios intereses incidirán positiva o negativamente sobre la organización. Por último, y quizás contando con un mayor poder sobre la actuación de las ONGD, se encuentran los gobiernos.

La actuación sobre estos grupos de interés únicamente implica un esfuerzo por conseguir con mayor eficiencia y mayor nivel de cumplimiento los objetivos fijados por la organización, sean cuales fueran éstos. Estos grupos

18 P. Kotler, D. Cámara e I. Grande (1995), p. 169. 
no constituyen los lobbies propios de la organización. Al contrario los lobbies seconstituyen como herramienta de relaciones públicas para ejercer presión sobre estos grupos de interés que detentan suficiente poder sobre la sociedad para bloquear o impulsar cualquier acción de la ONGD.

Se entiende por incidencia política el conjunto de acciones de información, diálogo, presión y/o denuncia (mediante movilización social, participación en órganos representativos, etc.) que las ONGD realizan, destinadas a personas e instituciones públicas, colectivos y entidades privadas con capacidad de decisión en aquello que afecta a las poblaciones del Sur, con la finalidad de influir de forma positiva en las relaciones entre los pueblos y en defensa de los colectivos más vulnerables de todo el mundo ${ }^{19}$.

Continuando con el análisis de la situación externa, habría que analizar las fuerzas no controlables del macroentorno que directa o indirectamente, y positiva o negativamente influyen sobre el área del Marketing. Es decir, hay que estudiar las grandes tendencias generales que afectan a la organización pero escapan a la influencia de la misma dentro del entorno demográfico, del entorno económico, del medio ambiente, del entorno tecnológico, del político legal o del cultural.

En cuanto al análisis interno de las debilidades y fortalezas, hay que centrar la atención en dos grandes bloques, los que se han denominado como políticas formales y funcionales. Es decir, por un lado se prestará atención a la identidad de la organización, y en especial al elemento diferenciador que aporta mayor valor para el mercado, aquello que realmente se sabe hacer bien. Y por otro lado, se contemplarán las distintas funciones de la organización: financiera, de recursos humanos,... y esencialmente la función de marketing, por la especial incidencia que ejerce sobre la planificación estratégica. En cada una de estas políticas se considerarán los recursos disponibles, las estrategias seguidas y la actuación desarrollada hasta ahora.

Una vez analizadas o identificadas las oportunidades, amenazas, fortalezas y debilidades procede evaluarlas de forma común, interrelacionándolas, para dar un diagnóstico de la situación. Para esta etapa, se utiliza generalmente lo que se conoce como matriz DAFO ${ }^{20}$. El proceso de elabo-

19 Manos Unidas (1998), p. 17.

20 Debilidades, Amenazas, Fortalezas y Oportunidades, también conocida como matriz SWOT (Strengths, Weaknesses, Opportunities \& Threats). 
ración de esta matriz consiste básicamente en la localización e identificación de las cuatro fuerzas comentadas para la organización en cuestión, valorándolas a partir de su posibilidad y su importancia es un instrumento de selección de aquellos aspectos realmente importantes sobre los que centrar la actuación estratégica de la organización. Este diagnóstico supone un esfuerzo de síntesis. Al hablar de la valoración de los distintos aspectos según su posibilidad se hace referencia a la probabilidad de que se manifiesten, mientras que, cuando se habla de la valoración según la importancia se hace referencia a la impresión subjetiva sobre las consecuencias en el cumplimiento de los objetivos de la organización, es decir, se valora el impacto. Una vez otorgada esta valoración, que nos permite seleccionar los elementos más relevantes para la organización, se analizarán conjuntamente todos los elementos, teniendo en cuenta que las fortalezas dirigen sus miras al aprovechamiento de las oportunidades, mientras que las debilidades deberían experimentar cierta mejoría para combatir las amenazas existentes.

En la realización de un diagnóstico corporativo será vital determinar la existencia de ventajas o desventajas competitivas. Si se cuenta con puntos fuertes en los que se es mejor que la competencia se habla de ventajas competitivas, que permitirán mantener la clientela, frente a la existencia de puntos débiles que además lo sean respecto a los competidores, que constituyen las desventajas competitivas.

Una vez concluido el análisis y diagnóstico de la situación la ONGD está en disposición de establecer sus prioridades estratégicas. 0 lo que es aún más:

El diagnóstico de la situación conduce directamente al planteamiento de las cuestiones o retos fundamentales que afectan a la organización, tanto globalmente como en determinados ámbitos (el de los usuarios, el de las políticas de financiación...).

Las prioridades estratégicas por definición, implican conflictos que se interponen en la relación de la organización con su entorno y con su dinámica de funcionamiento interno. El equipo planificador, por tanto, tiene que ser capaz de identificarlas, teniendo en cuenta su origen y sus consecuencias previsibles, y ordenarlas, en segundo momento, según el grado de intervención que merecen. Algunas prioridades estratégicas pueden llamar la atención inmediata, mientras que otras pueden no ser tan urgentes...

...en definitiva, el objeto de esta etapa es llamar la atención de la organización sobre algunos de los temas fundamentales para su supervivencia, prosperidad y eficacia. Precisamente, uno de los mayores beneficios que puede reportar a la organización el ejercicio de identificar las prioridades estratégicas es la posibilidad de pensar en términos de retos, en vez de pensar en términos de respuestas. Gracias al ejercicio de 
pensar las prioridades estratégicas, se ponen sobre la mesa muchos de los problemas que contaminaban el ambiente de algunos departamentos. De una vez por todas estos problemas se hacen «reales», se objetivizan e invitan a tomar partido ${ }^{21}$.

Una vez establecidas las prioridades estratégicas que determinarán los objetivos cuantificables en el ámbito corporativo, la organización pasará a la siguiente etapa en el proceso de planificación, es decir, buscará ¿qué hacer para conseguir los objetivos planteados? Es el momento de formular las líneas de acción concretas, y plasmarlo to do ello en un plan estratégico, por escrito. El diseño y culminación de estas estrategias y de las prioridades estratégicas, se encuentra directamente vinculado a la visión de la organización; existe una especie de retroalimentación entre todos estos elementos, tal y como se vio en la Figura 2. Si no existe una visión claramente definida, una vez se hayan diseñado las estrategias, ha llegado el momento de elaborar una descripción clara y precisa de dónde llegará la organización si se desarrollan exitosamente dichas estrategias. Es decir, se está en disposición de crear una frase motivadora que indique hacia dónde quiere llegar la organización.

En esta etapa se ha hablado de la necesidad de formular estrategias. Es decir, una vez enunciadas las prioridades estratégicas y convertidas en objetivos medibles hay que formular unas líneas de actuación. Para esta formulación se considerarán además todos los elementos que se han contemplado hasta el momento en el proceso de planificación. Todo el proceso de pensamiento estratégico que se desarrolle debe recogerse en un documento escrito que se conoce como plan estratégico. En este documento deben incluirse también los estándares de control que se establezcan y el procedimiento de control y seguimiento que tendrá lugar, siempre y cuando se contemplen las acciones concretas a desarrollar por cada individuo o grupos de individuos, junto al presupuesto y recursos humanos necesarios en cada caso. La extensión y el nivel de profundidad con el que tratar los distintos apartados del informe depende en gran medida del tamaño de la organización; en cualquier caso se trata de un documento necesario y de gran valor para las ONGD.

21 A. Vernis, M. Iglesias y Otros (1998), p. 82. 


\section{Conclusiones}

Una ONGD debe planificar sea cual sea su tamaño, sólo variará la complejidad del proceso a seguir, a pesar de que existen una serie de frenos o barreras iniciales que dificulten su puesta en práctica. La planificación debe contemplar aspectos globales, como la misión, visión y valores y aspectos concretos de las estrategias y acciones a desarrollar, para todo lo cual necesita como elemento indispensable contar con información suficiente.

La planificación, tal y como ocurre en el sector lucrativo, le aporta una serie de ventajas a la organización, tales como: sistematizar el pensamiento sobre el futuro, permite reaccionar más adecuadamente ante los cambios e identificar oportunidades de mejora, establece responsables, plazos y medidas de control y evaluación de forma que se coordina a todos los miembros de la organización.

Compartir la filosofía del Marketing basada en las relaciones de intercambio, a lo largo de todo el proceso de planificación en la labor desarrollada en el Norte, favorecerá el cumplimiento de los objetivos perseguidos por la ONGD. Teniendo en consideración la existencia de una doble relación de intercambio, se perseguirá de principio a fin la satisfacción de las distintas partes, sin obviar en ningún momento la misión de la ONGD cuyo cumplimiento se constituye como la propia satisfacción de la organización. De esta forma, manteniendo siempre presente al adoptante objetivo en su papel de cliente de la ONGD, la actividad en el Norte tendrá lugar de forma más eficaz y eficiente, consiguiendo maximizar los recursos disponibles.

\section{Bibliografía}

Albrecht, K. (1996), La misión de la empresa, Barcelona, Paidós Ibérica, 223 págs.

Assael, H. (1993) , Marketing: Principles \& Strategy, Orlando, The Dryden Press Harcourt Brace College Publishers, 711 págs.

Bloom, N. y Novelul, W.D. (1981), "Problems and Challenges in Social Marketing", J ournal of Marketing, vol 45, pp 79-88.

DRUCKER, P. (1990), Managing the Non-profit Organization, Great Britain, Butterworth-Heinemann, 178 págs. 
Fox, K.F. y KotleR, P. (1980), "The Marketing of social Causes: The first 10 years", J ournal of Marketing, vol 44, pp 24-33.

García Izquierdo, B. (1996), "La Gestión de la información para la mejora del intercambio entre una ONG y sus financiadores y colaboradores privados", Boletín de Estudios Económicos, n158, pp 209-230.

Guiltinan, J.P. y Paul, G.W. (1994), Administración de Marketing, Santa Fé de Bogotá, McGraw-Hill, 576 págs.

Iríbar Bilbao, F. y Martínez Pereda, J. (1996), “CCómo podemos controlar la gestión desarrollada por nuestra ONG?", Boletín de Estudios Económicos, n-158, pp 245-260.

KotLer, P. (1982), Marketing for Nonprofit Organizations, New J ersey, Prentice Hall, 528 págs.

Kotler, P. y AndReAsen, A.R. (1996), Strategic Marketing for Non-profit Organizations, New J ersey, Prentice Hall, 632 págs.

Kotler, P.; Cámara, D. y Grande, I. (1995), Dirección de Marketing, Madrid, Prentice Hall, 851 págs.

KotLeR, P. y LeVy, S. (1969), "Broadening the concept of Marketing", J ournal of Marketing, vol 33, pp 10-15.

LAMBIN, J . (1995), Marketing Estratégico, Madrid, McGraw-Hill, 610 págs.

Manos UnIDAS (1998), Cuaderno de formación básica nำ. El mundo de las ONG, Madrid, Manos Unidas, 59 págs.

Martín Dávila, M.; Manera Bassa, J. y Pérez del Campo, E. (1997), Marketing fundamental, Madrid, McGraw-Hill, 396 págs.

Martínez Sánchez, J.L. (1998a), La imagen de las ONG de desarrollo, Madrid, IEPALA, 264 págs.

Martínez Sánchez, J.L. (1998b), "Publicidad para causas sociales: argumentos racionales vs. argumentos emocionales", Comunicación y Sociedad, Volumen XI, no1, pp 69-90.

Montero Simó, M. J . (2001), Marketing Social: Una aplicación al caso de Manos Unidas Campaña Contra el Hambre, Córdoba, Tesis Doctoral, 570 págs.

OLIns, W. (1991), Identidad Corporativa, Madrid, Celeste Ediciones, 224 págs

Ortega CARPIO, M.L. (1994), Las ONGD y la Crisis del desarrollo. Un análisis de 
la cooperación con Centroamérica, Madrid, IEPALA Editorial y Publicaciones ETEA, 333 págs.

Palom Izquierdo, F.J y Tort Raventós, LL. (1999), Dirección Estratégica en Organizaciones «Non-Profit», Barcelona, Gestión y Planificación Integral, S.A., 207 págs.

Pérez del Campo, E. (1994), El Marketing en las instituciones no lucrativas, Anuario J urídico y económico Esculariense, vol 27, pp 365-395.

PrIDE, W. M. (1997), Marketing: Conceptos y Estrategias, México, McGraw-Hill, 877 págs.

Sáinz de Vicuña Ancín, J.M. (1995), El plan de marketing en la práctica, Madrid, Esic Editorial, 348 págs.

Schoell, W.F. y Guiltinan, J.P. (1991), Mercadotecnia: Conceptos y prácticas modernas, México, Prentice Hall, 822 págs.

ShAPIRO, B.P. (1973), "Marketing for nonprofit organizations", Harvard Business Review, Sept-Oct, pp 123-132.

Solomon, M.R. y Stuart, E.W. (1997), Marketing, Real People, Real Choice, London, Prentice Hall, 774 págs.

Van Riel, C. (1997), Comunicación Corporativa, London, Prentice Hall, 244 págs.

Vernis, A.; Iglesias, M. y Otros(1998), La gestión de las Organizaciones no Lucrativas, Bilbao, Ediciones Deusto, 221 págs.

Villafañe, J. (1993), Imagen positiva. Gestión estratégica de la imagen de las empresas, Madrid, Ediciones Pirámide, 341 págs. 